\title{
High Resolution X-Ray Phase Contrast Imaging of Maturating Cartilage.
}

Hélène Labriet $^{1,2}$, Caroline Bissardon ${ }^{2,3}$, Hervé Mathieu ${ }^{4}$, Ilyas Khan ${ }^{5}$, Laurent Charlet ${ }^{3}$, Sébastien Bérujon $^{6}$, Sylvain Bohic ${ }^{2}$, Emmanuel Brun ${ }^{2 *}$

${ }^{1 .}$ Novitom, 3 avenue du Doyen Louis Weil 38000 Grenoble, France.

2. Rayonnnement Synchrotron et Recherche Médicale, Université Grenoble Alpes, Grenoble, France.

3. Institut des sciences de la terre, Grenoble France Université Grenoble Alpes, Grenoble, France.

4. IRMaGe, INSERM US-17, F-38042 Grenoble, France.

5. Swansea University Medical School, Swansea, Wales

${ }^{6 .}$ European Synchrotron Radiation Facility, 71 avenue des Martyrs 38000 Grenoble, France.

* Corresponding author, emmanuel.brun@inserm.fr

The need for improved imaging modalities able to detect the onset of bone and joint diseases such as osteoarthritis is more than ever felt. Osteoarthritis, the progressive loss of articular cartilage and bony outgrowths in the joint, is the leading global cause of long-term musculoskeletal disability and chronic pain with hundreds of millions of people affected worldwide [1]. Current clinical and preclinical imaging modalities employed for joint disease diagnostics still present major limitations [2-3] especially regarding the detection of early cartilage and bony changes. For instance, the performances of X-ray absorption based micro Computed Tomography $(\mu \mathrm{CT})$ limit its use to bone defects and indirect imaging of cartilage. Magnetic Resonance Imaging (MRI), for its part, struggles to precisely render bony changes and microcalcifications that may occur during the first stages of diseases. The ability to co-image bones and cartilages, and in particular the constituent cells of cartilage, chondrocytes, is crucial. As a matter of fact, a disorganization of the pseudostratified morphology of the tissue is one of the hallmarks of the earliest phases of diseases. In order to study various imaging modalities, we used a simple and rapid (21 days) model of in vitro maturation of articular cartilage. In vitro maturation represents an ideal tool to develop proof-of-concept imaging modalities since it can generate large changes in tissue, cellular organization and structure within tissues where these processes naturally occur over time scale ranging from months to years [4].

With X-rays, the refraction index of materials can be a thousand times greater than its counterpart absorption factor for light elements [5]. This translates into a much greater contrast for soft tissues with X-ray imaging methods based on the sensing of the phase (so-called Phase Contrast Imaging (PCI)) in comparison to the current most traditional method based on absorption. For cartilage, PCI offers the great advantage of being sensitive to many tissues that constitute a joint. It allows the simultaneous visualization of both the high absorbing tissues like bones, and, also the less absorbing tissues such as fibrous cartilages, ligaments, tendons meniscus and associated soft tissues (synovial membranes and muscle) [6].

We introduce here the first extensive comparison for soft and hard tissue visualization, between PCI and the other full-field imaging modalities available for microscopic study of bone and cartilage. In addition to PCI, we imaged samples using high Resolution MRI, conventional $\mu \mathrm{CT}$, Optical Coherence Tomography (OCT). The PCI experiment was conducted at the beamline BM05 of the European Synchrotron Radiation Facility (ESRF), Grenoble, France. The sample to detector distance was set to 2.5 $\mathrm{m}$ and the X-ray photon energy was set to $17 \mathrm{keV}$ using a double Silicon crystal system within a BraggBragg geometry. The imaging detector was a scientific CMOS camera (PCO, Germany) mounted on an optic with a resulting pixel size of $3.5 \mu \mathrm{m}$. The MRI experiment was carried out at the IRMage MRI Facility (Grenoble, France) on a 9.4T MRI machine (Bruker 94/20 USR, AVIII-HD, Bruker, Ettlingen , Germany) with a 4 channels reception cryo-probe. We used a TurboRare-3D sequence (TR $=2200 \mathrm{~ms}$, $\mathrm{TE}=22 \mathrm{~ms}$, Rare factor $=4$ ) with a final isotropic pixel size of $50 \mu \mathrm{m}$ with a total acquisition time of 
14h40 per sample. The OCT images were obtained at the Institut Langevin using a LLtech tomograph with a pixel size of $1 \mu \mathrm{m}$. The Micro CT experiments were performed on a homemade $\mu \mathrm{CT}$ machine based on a micro-focus source (source diameter $=2 \mu \mathrm{m}$, energy $=50 \mathrm{kvP}$, pixel size $3.5 \mu \mathrm{m}$ ). Finally, in order to validate the resulting contrasts, we applied standard histology (H\&E and Safranin-O stainings).

Regarding the samples, four cartilage explants were excised under sterile conditions from the medial condyle of the metacarpophalangeal joints from immature male bovine calves and grouped in adjacent pairs for experimental analysis. For each explant cultured in standard medium, the paired sample was culture in a medium supplemented with growth factors to induce maturation. The explants were cultured for 21 days with the medium being changed every other day. The explants were eventually fixed in formylsaline solution for 12 hours before being transferred to a phosphate buffered saline solution (stored at $\left.4^{\circ} \mathrm{C}\right)$.

Figure 1 shows results from high-resolution imaging of a single bovine cartilage explant where the level of resolution achieved is compared to state of the art MRI and PCI-CT. In the PCI-CT image, chondrocytes are clearly visible as black round structures embedded in the cartilage matrix as well as cells within calcified cartilage that bridges the cartilage-bone interface. These structures are not visible in MRI despite the long acquisition time. Cartilage canals [7] are visible for the first time in 3D both in MRI and PCI-CT but only in the PCI-CT images we manage to render the remaining blood vessels within those canals. In this study, we also observed a varying number of chondrocytes and a noticeable change in their distribution between the samples that were cultured under conditions that induce precocious maturation (not shown here due to page limitation). In these latter samples a difference in the thickness and the composition of the cartilage canals was also evident. This work was thus a proof of concept that such experiments could be performed on small animals for long-term follow-up studies since both the radiation dose and the acquisition time can be made mutually compatible.

\section{References:}

[1] R. Bitton, Am. J. Manag. Care, vol. 15, no. 8 Suppl, pp. S230-5, Sep. 2009.

[2] J. L. Tremoleda et al, EJNMMI Res., vol. 1, no. 1, p. 11, Jul. 2011.

[3] D. Hayashi et al, Br. J. Radiol., p. 20170349, Jan. 2018.

[4] I.M. Khan et al Arthritis Rheum. 63(11):3417-27 Nov. 2011

[5] A. Bravin et al, Phys. Med. Biol., vol. 58, no. 1, pp. R1-R35, Jan. 2013.

[6] A. Horng et al,Invest. Radiol., vol. 49, no. 9, pp. 627-34, Sep. 2014.

[7] A. M. Lutfi, J. Anat., vol. 106, no. Pt 1, pp. 135-45, Jan. 1970.
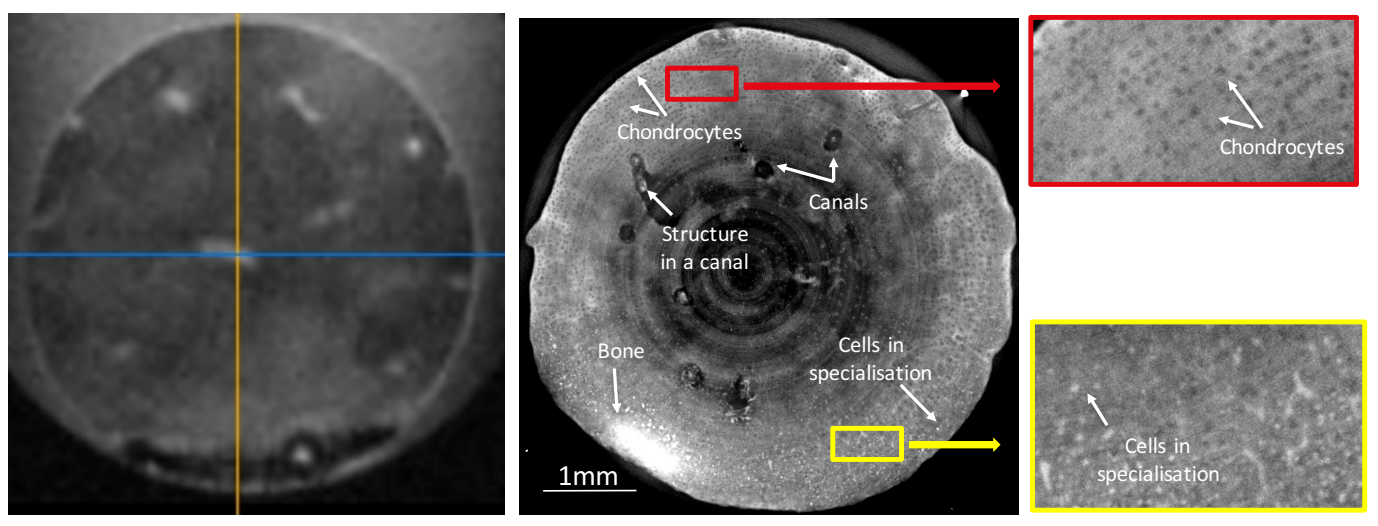

Figure 1. High resolution of maturating cartilage. MRI slice (left) obtained with a $9.4 \mathrm{~T}$ using a cryo probe. PCI-CT slice (right) and insets of the same cartilage explant. The different visible structures are indicated by arrows. A clear difference can be noted in between the two different images 